\title{
PHOTOVOLTAIC EFFECT WITH A SPECTRALLY DEPENDENT SIGN IN TYPE-II GaAlAs HETEROSTRUCTURE
}

\author{
M. JEżEWSKI \\ Institute of Experimental Physics, Warsaw University \\ Hoża 69, 00-681 Warszawa, Poland \\ R. Teissier, F. Mollot and R. Planel \\ Laboratoire de Microstructures et de Microelectronique, CNRS \\ 196, Av. H. Ravera, 92220 Bagneux, France
}

\begin{abstract}
An asymmetric $n-i-n$ type-II GaAs/GaAlAs/AlAs quantum heterostructure was designed and fabricated in order to observe a static photovoltage with a spectrally dependent sign. This photovoltage is associated with a light induced spatial separation of electrons and holes within selected regions of the structure. The observed photovoltage spectrum is compared with luminescence and luminescence excitation results.
\end{abstract}

PACS numbers: 78.55.Cr, 72.40.+w, 72.20.Jv

A type-II structure can be created within GaAlAs system by combining a thin layer of $\mathrm{GaAs}$ with a layer of $\mathrm{Ga}_{1-x} \mathrm{Al}_{x} \mathrm{As}$ ( for $x>0.35$ ). This is the simplest structure where a $\mathrm{Ga}_{1-x} \mathrm{Al}_{x} \mathrm{As}$ layer, trapping electrons in $X$ conduction state $[1,2]$, is adjacent to a GaAs layer serving as a hole collector. It is possible to separate hole and electron wells by inserting between them a third material playing a role of an effective barrier for both types of carriers. The spatial separation of electron and hole accumulation regions leads to the creation of an internal electric field acting on a region lying in between. Such a field modifies an electronic structure of the sample $[3,4]$ and is associated with a potential drop across the structure. As a consequence, under illumination, one can observe an external photovoltage appearing at the extremities of the microstructure. In a simple type-II structure, like one period of superlattice, the sign of such a photovoltage should be spectrally independent, because the carrier separation takes place always in one direction (holes to GaAs, electrons to GaAlAs alloy). This paper describes the microstructure where a photovoltage with a spectrally dependent sign can be detected.

The $n-i-n$ structure made within GaAlAs system was fabricated by molecular beam epitaxy. The sequence of central layers of microstructure is designed in Fig. 1. Two AlAs layers (LE and RE) of width of $5 \mathrm{~nm}$ are separated by large 
barriers from a central, $2 \mathrm{~nm}$ thick GaAs layer (H). Left barrier (LB), made as GaAs/AlAs pseudoalloy (with mean Al content of 0.33 ), has a thickness of $60 \mathrm{~nm}$. Right barrier (RB) is made from $15 \mathrm{~nm}$ thick $\mathrm{Ga}_{0.75} \mathrm{Al}_{0.25} \mathrm{As}$. Such combination of layers is embedded in GaAs/AlAs pseudoalloy with mean Al content varying from $50 \%$ to $0 \%$ and changing $\mathrm{Si}$ doping with the aim to transit smoothly from the central part of the structure to $n^{+} \mathrm{GaAs}$ buffer and the cap layer. In the

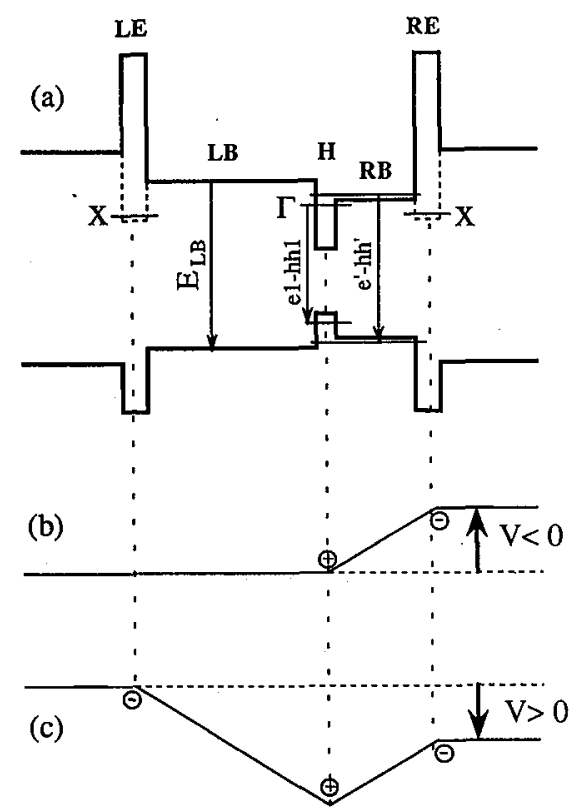

Fig. 1. Scheme of effective potentials for electrons (solid line in gamma point and dashed line in $X$ point) and holes along the structure (a). Calculated energies of confined states are also sketched for electrons and heavy holes. Expected electrostatic potential and photovoltage for excitation energies lower (b) and higher (c) than left barrier (LB) energy gap.

fabricated structure GaAs/AlAs pseudoalloys were used for technological reasons. However, it is possible to replace them by simple GaAlAs alloys with appropriate Al content. The $300 \mu \mathrm{m} \times 300 \mu \mathrm{m}$ MESA structures were made by standard photolithography and chemical etching and $\mathrm{Au}-\mathrm{Ge}-\mathrm{Ni}$ ohmic contacts with a central window were alloyed. Luminescence, luminescence excitation and photovoltage spectra were measured at $T=10 \mathrm{~K}$ by means of conventional experimental setup with DCM Dye excitation laser.

The luminescence spectrum (Fig. 2) consists of two main peaks at the energies of $1.95 \mathrm{eV}$ and $1.78 \mathrm{eV}$. They can be interpreted as e1-hh1 transition within left barrier (LB) and e1-hh1 recombination in central GaAs well (H). In the excitation spectrum of the central well luminescence (Fig. 2) one can distinguish peaks corresponding to hh1-e1 and lh1-e1 transition of central GaAs well, hh1-e1 and lh1-el transition within right $\mathrm{Ga}_{0.75} \mathrm{Al}_{0.25} \mathrm{As}$ barrier and the rise of excitation due 
to absorption within left GaAs/AlAs barrier. All experimentally found energies agree well with the energies obtained from transfer matrix [5-7] calculations.

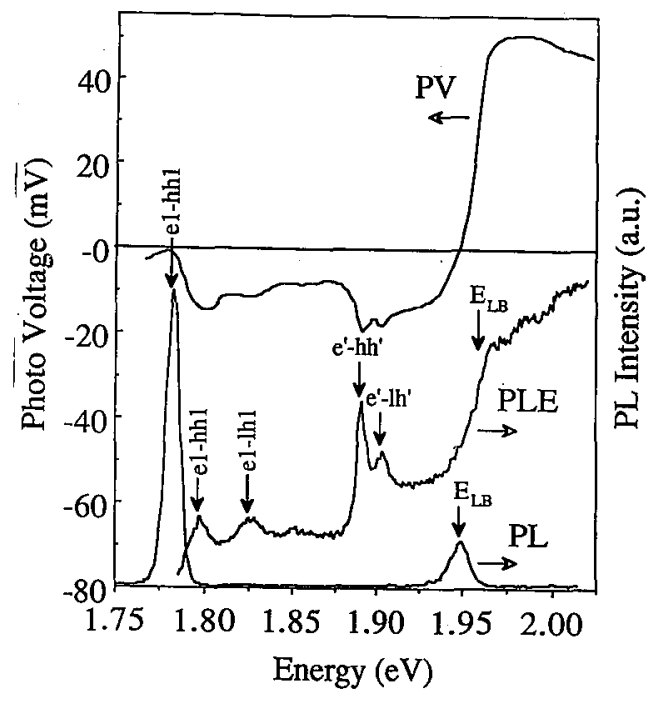

Fig. 2. Photoluminescence (PL), photoluminescence excitation (PLE) and photovoltage (PV) spectra performed at $T=10 \mathrm{~K}$. PL and PLE were obtained on as-grown sample. The PV spectrum was measured on a MESA structure loaded by a $1 \mathrm{G} \Omega$ resistance, with an incident light intensity of $200 \mathrm{~W} / \mathrm{cm}^{2}$.

The open-circuit photovoltage spectrum is presented in Fig. 2. Below the energy of the lowest electron transition in the structure (e1-hh1 transition in GaAs layer) the measured photovoltage is negligible. For incident light energies greater than this energy one can observe a steep increase in a negative photovoltage due to accumulation of electrons in the right AlAs layer while holes remain in the GaAs well. Such a transfer of electrons from the central well to the AlAs layer is possible because of high electron transfer probability through the $\mathrm{Ga}_{0.75} \mathrm{Al}_{0.25} \mathrm{As}$ barrier which has a relatively low height for strongly confined electrons in GaAs. The negative photovoltage increases further when the incident photon energy reaches the right $\mathrm{Ga}_{0.75} \mathrm{Al}_{0.25} \mathrm{As}$ barrier energy allowing the excitation of mobile carriers in a wider region of the structure. For high excitation powers this second increase in the photovoltage does not take place, as it is already saturated for lower photon energies. In the photovoltage spectrum one can distinguish all relevant details corresponding to that of luminescence and excitation spectra. The measured photovoltage decreases rapidly as the incident photon energy reaches the fundamental energy of the left GaAs/AlAs barrier, indicating that the electron accumulation in left AlAs layer takes place. By further increase in the excitation energy, a change of sign of the measured photovoltage is possible. The positive potential rise across the left barrier associated with electron accumulation in the left AlAs layer prevails on the negative potential drop across the right barrier observed before. The 
reason for this could be more effective absorption of photons (excitation of electrons) in the left barrier, being larger than the right one, and a subsequent greater accumulation of electrons in the left AlAs layer. At the same time, even for smaller electron concentration accumulated in this layer, the resulting left electric dipole can be greater than the right one because it has greater length (left barrier is four times larger than the right one). The measured photovoltage is likely a result of interplay between the above two mechanisms, as it depends on a photon energy, exciting laser power and load resistance of the sample.

In conclusion, we have demonstrated a type-II structure specially designed in order to obtain a static photovoltage with a spectrally dependent sign. By cascading more structures like the one described above, it should be possible to obtain a sample in which potential drops in each period add, resulting in a high static photovoltage measured for some incident photon energies. Such a structures could find a possible applications as, for example, 3-state logic elements or detectors of radiation with particular wavelengths.

\section{References}

[1] E. Finkman, M.D. Sturge, M.C. Tamargo, Appl. Phys. Lett. 49, 1299 (1986).

[2] G. Danan, B. Etienne, F. Mollot, R. Planel, A.-M. Jean-Louis, F. Alexandre, G. Le Roux, J.-Y. Marzin, H. Savary, B. Sermage, Phys. Rev. B 35, 6207 (1987).

[3] M. Jeżewski, F. Mollot, R. Planel, Appl. Phys. Lett. 56, 2422 (1990).

[4] M. Jeżewski, R. Teissier, F. Mollot, R. Planel, Superlattices Microstruct. 8, 329 (1990).

[5] G. Bastard, Phys. Rev. B 24, 5693 (1981).

[6] G. Bastard, Phys. Rev. B 25, 7584 (1982).

[7] Perng-fei Yuh, K.L. Wang, Phys. Rev. B 38, 13307 (1988). 